\title{
Chemistry and Transport Effects on Critical Flame Initiation Radius for Alkanes and Aromatic Fuels
}

\author{
Jeffrey S. Santner, Sang Hee Won, and Yiguang Ju \\ Department of Mechanical and Aerospace Engineering \\ Princeton University, Princeton, NJ 08544, USA \\ *Corresponding Author \\ Jeffrey S. Santner, Ph.D. \\ 9700 S Cass Avenue \\ Lemont, IL 60439 \\ Email: jsantner@anl.gov
}

\section{Colloquium}

\section{Laminar Flames}

Keywords: Critical radius; Transport-weighted enthalpy; Radical index; Outwardly propagating spherical premixed flame

\begin{tabular}{lr}
\multicolumn{2}{c}{ Word Counts } \\
Total & $\mathbf{5 7 7 6}$ \\
Main text & 3798 \\
Equations & 53 \\
References & 577 \\
Figure 1 & 104 \\
Figure 2 & 173 \\
Figure 3 & 168 \\
Figure 4 & 172 \\
Figure 5 & 252 \\
Figure 6 & 159 \\
Figure 7 & 166 \\
Figure 8 & 154
\end{tabular}




\title{
Chemistry and Transport Effects on Critical Flame Initiation Radius for Alkanes and Aromatic Fuels
}

\author{
Jeffrey S. Santner, Sang Hee Won, and Yiguang Ju \\ Department of Mechanical and Aerospace Engineering \\ Princeton University, Princeton, NJ 08544
}

\begin{abstract}
The combined effects of transport and fuel reactivity on the critical flame initiation radius for nalkanes and aromatics are investigated experimentally and numerically using outwardly propagating spherical flames at atmospheric pressure. The effects of transport on the critical flame initiation radius are examined by comparing n-alkanes with different sizes (n-heptane vs. n-decane) but similar molecular structure and reactivity. The effect of fuel reactivity and chemistry on the flame initiation radius are investigated by comparing two alkylated benzene isomers, 1,3,5-trimethylbenzene and n-propylbenzene, which have distinctive reactivity and molecular structures but have the same molecular size and transport properties. The results showed that for n-alkanes, consistent with theoretical predictions, the effect of transport on the critical flame initiation is small for large hydrocarbon fuels. For aromatic fuels, it is found that fuel reactivity defined by fuel molecular structure has a significant impact on the critical flame initiation radius. This observation also supports the theoretical prediction. The results show that fuel with lower reactivity (1,3,5-trimethylbenzene) has a larger critical radius than that with higher reactivity (n-propylbenzene). It is also demonstrated that there is a linear correlation between the critical strain rate and the peak in-flame $\mathrm{OH}$ concentration. A radical index combined with transport-weighted enthalpy is proposed to correlate the critical radius of all tested alkanes and aromatic fuels. It is found that the critical radius of all fuels can be reasonably correlated to the product of the radical index and the transport-weighted enthalpy. The results reveal that, similar to flame extinction limits, the critical radius is subject to the combined effect of enthalpy transport and fuel reactivity. The present results suggest that an increase of the product of the transport weighted enthalpy and fuel reactivity may lead to lower chance of ignition failure in internal combustion engines and turbine engine relight.
\end{abstract}




\section{Introduction}

Ignition failure remains to be a big challenge for small scale lean burn internal combustion engines [1-3] and high altitude gas turbine relight [4]. Many experimental [5-8], theoretical, and computational studies [5, 9-11] have investigated factors controlling successful flame initiation. Recent theory and simulations $[10,11]$ clearly showed that the minimum ignition energy (MIE) is governed by a critical radius below which an initial ignition kernel will quench, thus flame initiation fails, even for a fuel mixture above the flammability limit. The theory [11] also suggested that the critical flame radius and the MIE increase rapidly with the increase of the Lewis number (or the size of fuel molecules) when the mixture Lewis number is below 1.5, whereas further increasing Lewis number beyond 1.5, the increase of the critical radius becomes attenuated. Moreover, theory in $[5,11]$ showed that the critical flame initiation radius is strongly affected by the overall activation energy, representative of fuel chemistry. Nevertheless, few experiments have been carried out to demonstrate the effect of fuel chemistry on the critical radius or to verify the proposed theories.

The existence of a critical radius for successful flame initiation has been experimentally observed for hydrogen, methane, and other small hydrocarbon flames [5, 6, 10, 12, 13] in spherical combustion chambers. The impact of the critical radius on flame initiation has been also experimentally observed even in a high speed turbulent flow when the premixed fuel/air mixtures were ignited using repetitive nano-second pulsed discharge [14]. At the same ignition energy, the results demonstrate that the formation of a single large ignition kernel greater than the critical radius using multiple overlapping pulse discharges is necessary for successful ignition. It also shows that the ignition improvement from plasma discharge is stronger for methane than for ethylene, indicating the impact of fuel chemistry in the ignition process. A recent study [8] on the effects of pressure found that the critical radius of $n$-decane increases rapidly with the decrease of pressure and became greater than $1 \mathrm{~cm}$ at $1 \mathrm{~atm}$, consistent with theoretical predictions $[5,11]$. The large critical radius at reduced pressure may cause ignition failure for high altitude engine relight. Although the aforementioned studies have focused on single-component fuels, the critical radius of real transportation fuels has not been fully understood due to its complex chemical composition, exhibiting a broad range of molecular structures and sizes. Recent studies in formulating surrogate fuel mixtures for petroleum-derived and alternative jet fuels [15-17] have shown that the molecular size and structure of the fuel components significantly modifies combustion behavior [18, 19]. Therefore, it is important to understand the kinetic effect of fuel chemistry on the critical ignition radius, especially for large hydrocarbon fuels relevant to real transportation fuels.

Theoretically, the critical radius is governed by the flame extinction subject to strong stretch rate (curvature). The effects of transport and chemistry on flame extinction have been extensively studied [18-21]. A recent experimental study [18] of diffusion flame extinction of various hydrocarbon fuels with different molecular structures showed that the extinction limit of a diffusion flame can be correlated into a linear relation with the transport-weighted enthalpy and the radical index. It was also shown that the fuel reactivity and radical pool concentration have significant impact the extinction limits. The increase of fuel molecular size dramatically reduces the extinction limit of diffusion flames due to the slower diffusion of fuel molecules to the reaction zone. It is of interest to investigate whether these findings in diffusion flames are applicable to the extinction of premixed flames, because premixed flames are much less affected 
directly by the fuel transport properties and more affected by fuel chemistry due to fuel oxidation in the preheating zone. To the authors' knowledge no experimental reports are available on the effects of transport and fuel chemistry on the critical flame radius, especially for large alkanes and aromatic fuels [8]. As such, it is not clear how the critical flame radius for flame initiation is affected by fuel molecular size, structures, and reactivity.

The objective of this paper is to investigate the impacts of transport and fuel chemistry on the critical flame radius using both n-alkanes and aromatic fuels in outwardly propagating spherical flames. First, the effect of transport properties on the critical radius is studied by using two different sizes of n-alkanes (n-heptane and n-decane), which share identical high temperature reactivities [18]. Second, the effect of fuel chemistry on the critical radius is investigated by comparing the critical radius of two aromatic isomers, n-propylbenzene and 1,3,5trimethylbenzene, which have the same molecular size but different molecular structures and reactivity $[15,18]$. Thirdly, the critical radii of all four fuels are numerically modeled and compared with experimental data. Finally, the effects of transport, molecular structure, and fuel reactivity on the critical radius are examined by correlating the critical radius with the transport weighted enthalpy and radical index for all four fuels.

\section{Experimental and Numerical Methods}

Critical radius measurements are conducted in a constant volume spherical chamber housed in a PID controlled oven (Fig. 1) [8]. Details of experiments have been reported elsewhere [8]. The spherical chamber has a radius of $10 \mathrm{~cm}$ and has two optical windows for schlieren imaging. A fan is installed inside the oven to ensure that the temperature non-uniformity is below $5 \mathrm{~K}$. Two nalkanes and two aromatic isomers are tested; ndecane (nC10), n-heptane (nC7), n-propylbenzene (nPB), and 1,3,5-trimethylbenzene (135TMB). The liquid fuels are pre-vaporized and transferred into the spherical chamber via a heated line and needle valve. The fuel vaporization chamber,

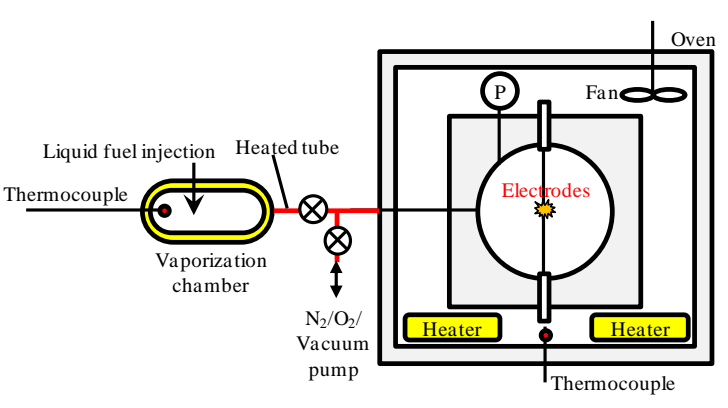

Figure 1. Schematic of experiment. oven, spherical chamber, and connecting tubing are all maintained at $400 \mathrm{~K}$ with three separate PID controlled resistance heaters, monitoring the temperatures with K-type thermocouples. The fuel-air mixtures are prepared using the partial pressure method. The combustion chamber pressure is measured by using a pressure gauge (Kulite XTEL-190) during mixture preparation. To ensure no condensation of liquid fuel in the tubing, the pressure gauge is also housed in the oven. The mixture is allowed ten minutes to become quiescent and complete mixing. The mixture is ignited at the center of the chamber using a spark system with an ignition coil. The flame front location and flame surface smoothness (to avoid the effect of flame instability) are recorded during flame propagation using a high-speed Schlieren imaging system. The main uncertainty for the experiments originates from the fuel concentration for lean mixtures using the fuel partial pressure $(1 \%)$. In order to increase the fuel partial pressure and reduce this source of uncertainty, a high pressure (e.g. $5 \mathrm{~atm}$ ) fuel and air mixture is created and then the mixture is bled into a vacuum until the desired experimental pressure is reached. 
The flame front location as a function of time is extracted from the Schlieren images by using an in-house edge finding program. To minimize the uncertainty from non-spherical flame shape caused by the electrodes, the flame radius is determined by using a circle-fitting algorithm. The time history of the flame front location is smoothed by using a second-order polynomial fitting in a $2 \mathrm{~mm}$ interval. The flame speed can be extracted by using a linear extrapolation method [22]. For the measurement of the critical radius, the flame front trajectory is plotted as function of flame strain rate [8]. The critical radius in this study is defined by the location separating the unsteady flame transition (Regime II) from the normal flame propagation (Regime III), consistent with the definition used in [8]. Theory $[5,11]$ defines the critical radius at the location between the ignition kernel propagation (Regime I) and Regime II, where flame trajectories with different ignition energies converge onto a single trajectory. However, this point is difficult to obtain experimentally.

Simulations of the spherical flame propagation are conducted using A-SURF [9]. A $2 \mathrm{~mm}$ radius hot spot at $1600 \mathrm{~K}$ is used to initiate the flame. The temperature profile of the hot spot does not affect the determination of the critical radius or flame speed, as the flame trajectory collapses onto a single manifold at radii larger than the critical radius regardless of the initial conditions [5, 11]. The computational domain is $10 \mathrm{~cm}$ in radius with reflective boundary conditions both at the center and at the wall. To resolve the flame front, a six level mesh refinement with minimum grid size around 10 microns is used. A-SURF solves the conservation equations of a compressible reactive flow system. The accuracy of A-SURF for flame simulation has been extensively validated [9]. The critical radius in simulations is found in the same manner as the experiment. The kinetic models of $\mathrm{nC} 7$ and $\mathrm{nC} 10$ from the Lawrence Livermore National Laboratory $[23,24]$ (LLNL) are adopted. For modeling nPB and 135TMB flames, the aromatic models developed at Princeton University $[19,25]$ are used. All models are systematically reduced for high temperature flames using the multi-generation path flux analysis method [26], resulting in, 72 species for $\mathrm{nC} 10,57$ species for $\mathrm{nC}$, 163 species for $135 \mathrm{TMB}$, and 107 species for $n P B$.

\section{Results and Discussion}

The time histories of flame front locations of four different fuel-air mixtures at equivalence ratio $(\varphi)$ varying from 0.7 to 1.0 have been measured and the flame propagation speeds $\left(S_{b}\right)$ are analyzed in a function of stretch rate $(K)$. Figure 2 compares the flame trajectories $\left(S_{b}\right.$ vs $\left.K\right)$ at $\varphi$ $=0.7$ for four fuels. It is clearly seen that for all four fuel air mixtures, there exist three different flame regimes: the ignition kernel propagation regime (I), the unsteady flame transition regime (II), and the steady flame propagation regime (III). The critical flame radius defined here is the location which separates regime II from regime III. The distinct three regimes are observed for all

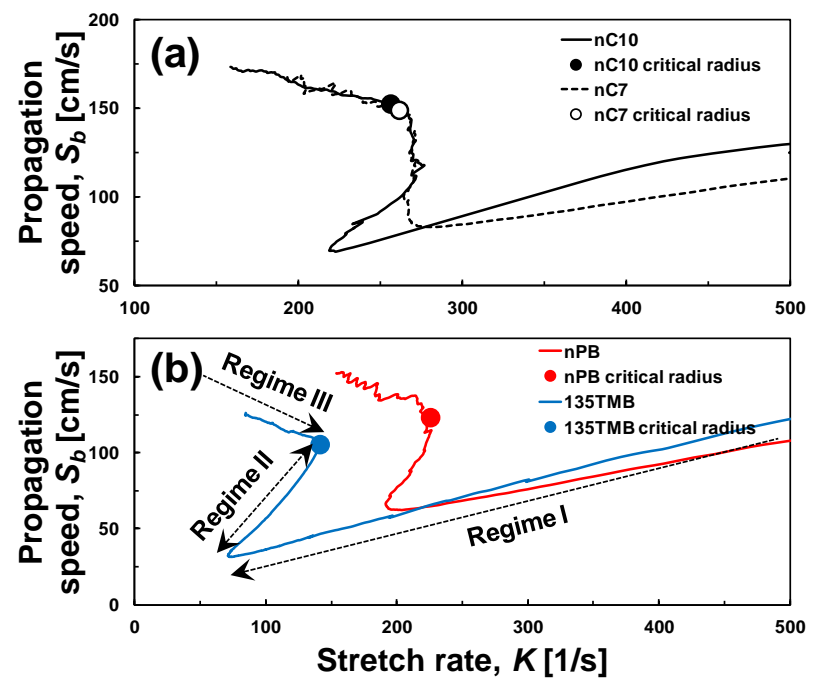

Figure 2. Comparisons of flame trajectories, changes of flame propagation speed $\left(\mathrm{S}_{\mathrm{b}}\right)$ with stretch rate $(\mathrm{K})$ for $(\mathrm{a})$ $\mathrm{nC} 10$ and $\mathrm{nC} 7$ and for (b) $\mathrm{nPB}$ and 135TMB. 
equivalence ratios of lean mixtures $(0.7 \leq \varphi \leq 1.0)$. Figure $2 \mathrm{a}$ shows that for similar fuel reactivity and oxidation chemistry, the critical radii of $\mathrm{nC} 7$ and $\mathrm{nC} 10$ are almost the same, even though their molecular weights are about $40 \%$ different, consistent with the previous theoretical prediction $[5,11]$. Due to the nonlinear effect of Lewis number $(L e)$ on flame propagation, the theory predicted that for $L e$ less than 1.5, the increase of $L e$ greatly increases the critical radius. However, for $L e>1.5$, the increase of the critical radius with $L e$ becomes attenuated. Here the values of effective Le have been calculated by the method in [27]. Since the lean n-heptane and n-decane/air mixtures both have $L e$ greater than 1.8, the increase of fuel molecular size only has small impact on the critical radius. Considering that both $\mathrm{nC} 7$ and $\mathrm{nC} 10$ have identical high temperature oxidation chemistry, forming similar intermediate species (mainly $\mathrm{C}_{2} \mathrm{H}_{4}$ ), the difference of the effective Le among $\mathrm{nC} 7$ and $\mathrm{nC} 10$ can be regarded even smaller than the difference in fuel molecular size [18]. Therefore, it can be concluded that for large n-alkane fuels with similar fuel reactivity, the transport effect on the critical flame initiation radius is negligible.

However, Figure $2 \mathrm{~b}$ shows that with the same fuel molecular size but different fuel reactivity, $\mathrm{nPB}$ has a much smaller critical radius (higher critical strain rate) than that of 135TMB. This is because 135TMB has a lower fuel reactivity or radical index [18] than n-PB due to the difference in molecular structure. As a result, 135TMB requires a larger critical radius (lower critical strain rate) to successful transition from the initial ignition kernel propagation regime (I) to the subsequent the unsteady flame transition regime (II) and normal flame regime (III), indicating that 135TMB needs larger MIE for successful flame initiation. This result is also consistent qualitatively with the theoretical prediction $[5,11]$ based on global activation energy. Therefore, it can be concluded that for large hydrocarbon fuels, fuel reactivity governed by the molecular structure has a significant impact on the critical radius. A fuel with lower fuel reactivity requires a large critical radius or MIE for successful ignition.

To understand the detailed mechanism of the impact of fuel chemistry on the critical radius, the critical radius and critical strain rates of all four fuels have been measured and numerically modeled at different equivalence ratios. The critical strain rate is defined here as the strain rate at the point separating regime II from regime III. The comparisons between experimental measurements and simulations are shown in Figs. 3 and 4, respectively. It is seen in Fig. 3 that the critical radius decreases as the equivalence ratio increases. This is because the effective Lewis number of the fuel/air mixtures decreases as the equivalence ratio increases. Experimental data show that on the fuel lean side, $\mathrm{nC} 7$ has almost the same critical radius as nC10. However, the difference between the critical radii of $\mathrm{nC} 7$ and $\mathrm{nC} 10$ increase as the equivalence ratio increases. This is because Le is decreasing with increase of $\varphi$ to 1.0, where Le effects becomes more important at $L e<1.5[5,11]$. At near stoichiometric condition, the critical radius of $\mathrm{nC} 7$ becomes smaller than $\mathrm{nC} 10$,

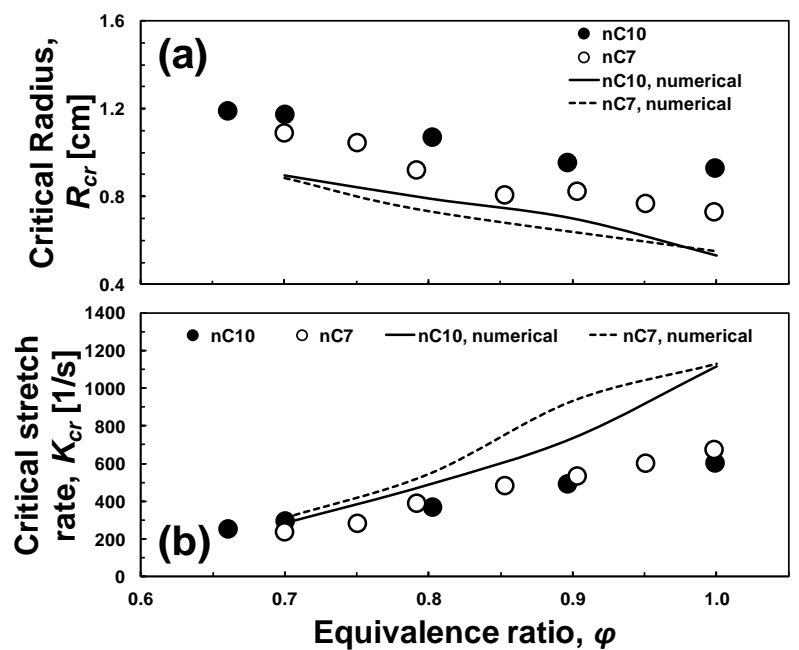

Figure 3. Comparisons between the measured and numerically computed (a) critical radius and (b) critical stretch rate as a function of equivalence ratio for $\mathrm{nC} 10$ and $\mathrm{nC7}$. 
indicating that the molecular size also plays a role on critical radius as the fuel/air mixture approaches to stoichiometric condition. Numerical predictions capture a similar trend as the experiments, but significantly underpredict the critical radii.

Figure $4 \mathrm{a}$ shows that $\mathrm{nPB}$ has smaller critical flame radii than $135 \mathrm{TMB}$ solely due to the effect of fuel chemistry. Similar to alkane fuels, the critical radius reduces rapidly as the equivalence ratio increases. The effects of fuel chemistry on the critical radius can be demonstrated more clearly by using the critical stretch rate. The critical stretch rate for $\mathrm{nPB}$ is a factor of two larger than for 135TMB due to its faster fuel oxidation chemistry. Again, numerical results predict similar trend of the effects of fuel chemistry and equivalence ratio but do not reproduce the measurements quantitatively.

The results shown in Figs. 3 and 4 demonstrate that the critical radius and stretch rate are mostly governed by fuel chemistry at fuel lean conditions. Furthermore, for near stoichiometric conditions, the critical radius is sensitive to both fuel molecular size and fuel chemistry. These results agree qualitatively with the theory $[5,11]$ and the extinction strain rates measured in premixed counterflow flames [28-30] at different conditions. 
To explore the relation between fuel reactivity and the critical radius, the dependence of flame propagation speed, flame thickness, the maximum heat release rates, and radical pool (summation of maximum $\mathrm{O}, \mathrm{H}$, and $\mathrm{OH}$ mole fractions) on flame stretch rate for the four fuel mixtures at equivalence ratio of 0.8 are plotted in Fig.5a-d, respectively. It is seen that the flame regimes strongly affect the flame propagation speed, thickness, heat release rate and the radical pool. For the ignition kernel propagation regime (I), the flame propagation speed, heat release rate and radial pool decrease rapidly while the flame thickness increases as the flame propagates outwardly (decrease of strain rate). At the transition from regimes I to II, the flame propagation speed, heat release rate, and radical pool reach local minimum, while the flame thickness reaches maximum. After passing this point, the flame structure changes and the flame speed increases during regime II, yielding a normal propagating flame (III) and a linear dependence of flame propagation speed on strain rate (Fig. 5a). Comparing the radical pool concentrations of the four fuels, it is clearly seen that 135TMB has the lowest fuel reactivity and thus the largest critical radius. In addition, Figure 5c shows that the fuel molecular structure and heating value also affect the maximum heat release rate and the flame speed as well as the critical radius. Note that $\mathrm{nPB}$ has a similar radical pool concentration to $\mathrm{nC} 10$, but exhibits lower maximum heat release rate. Therefore, in order to understand the dependence of critical radius, not only the fuel reactivity and molecular size but also the fuel heating value must be considered.
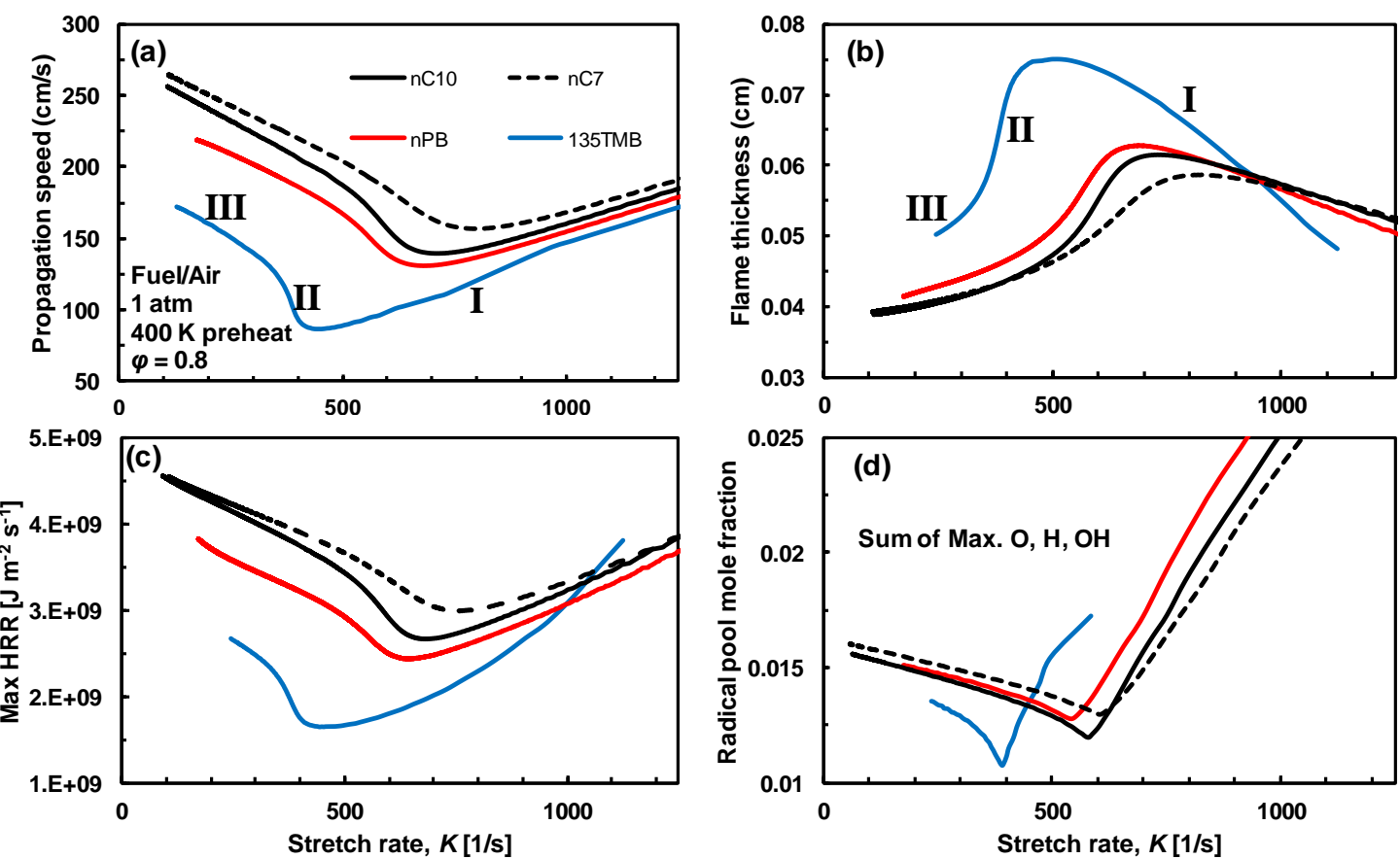

Figure 5. Comparisons of (a) flame propagation speed, (b) flame thickness, (c) maximum heat release rate (HRR), and (d) gross radical pool mole fraction among $\mathrm{nC10}, \mathrm{nC7}, \mathrm{nPB}$, and 135TMB from numerical calculations. 

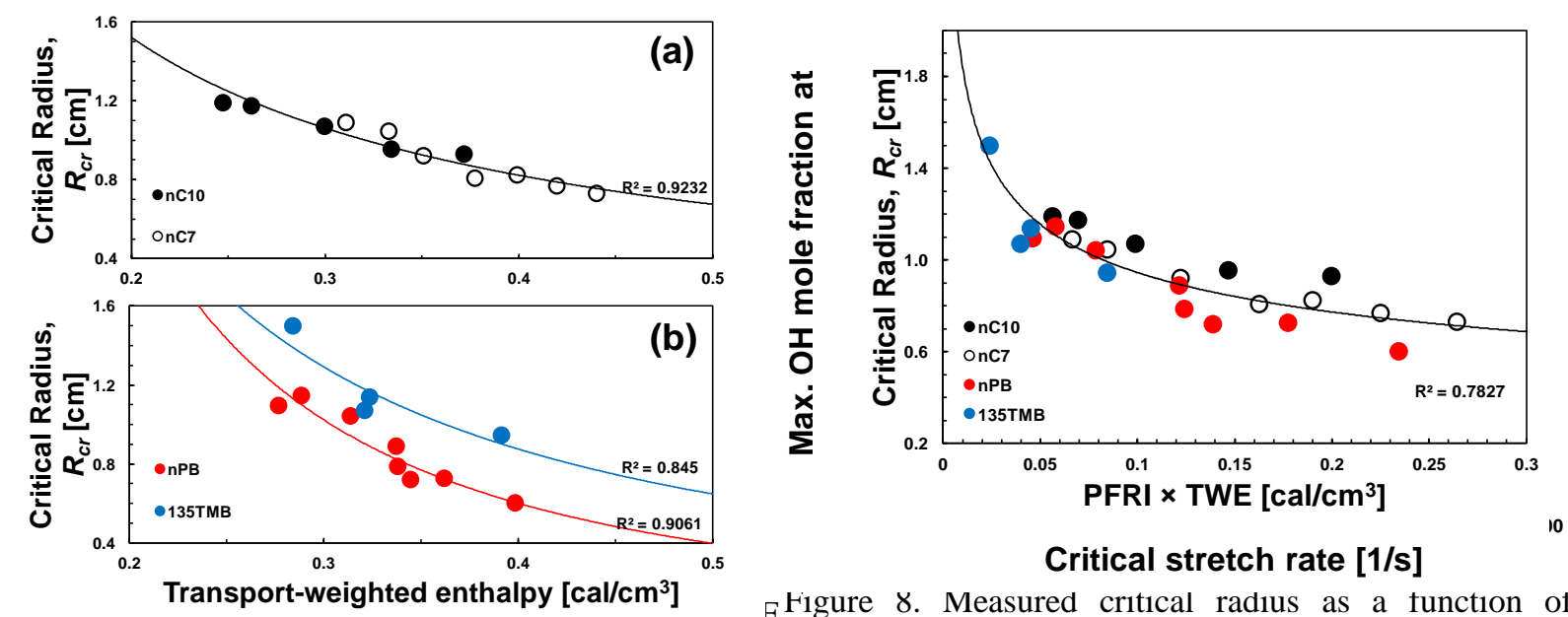

8. Measured critical radius as a function of Figure 7. Measured critical radius as a function of premixed flame radical index (PFRI) multiplied with

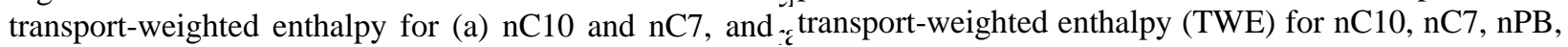
(b) nPB and 135TMB.

$r c$ and 135TMB.

To correlate the diffusion flame extinction limit with the fuel reactivity, transport properties and fuel heating value, Won and co-workers introduced the concepts of radical index and transport weight enthalpy and showed that there is a linear relation between the diffusion flame extinction limit and the product of the radical index and the transport weighted enthalpy $[18,31]$. We here attempt to use a similar concept to correlate the critical radius and strain rate of premixed flames to the transport and chemical reactivity of hydrocarbon fuels. In flames, $\mathrm{OH}$ is the dominant radical which governs the chemical heat release rate (e.g. $\mathrm{CO}+\mathrm{OH}=\mathrm{CO}_{2}+\mathrm{H}$ ). Since the radical pool mole fraction is an indication of fuel reactivity and has a significant impact on the critical flame radius and stretch rate (Figs. 3 and 4), we use the maximum $\mathrm{OH}$ concentration in the flame at the critical radius as the indicator of the radical pool. The dependence of the computed maximum $\mathrm{OH}$ concentration at the critical radius on the computed critical stretch rate is shown in Fig. 6. It is seen that there is a linear relationship between the maximum $\mathrm{OH}$ mole fraction and the critical strain rate. The good linear relation suggests that the maximum $\mathrm{OH}$ mole fraction can be used to scale the critical strain rate. Therefore, in this study, we define a normalized premixed flame radical index (PFRI) by using the product of the maximum $\mathrm{OH}$ mole fraction $\left(\mathrm{X}_{\mathrm{OH}}\right)$ and the strain rate $(\kappa)$ of a fuel scaled by that of $\mathrm{n}$-heptane:

$$
P F R I=\frac{\operatorname{Max} . O H_{\phi=1} \times K}{\left(\operatorname{Max} \cdot O H_{\phi=1} \times K\right)_{n C 7}}
$$

The product of the maximum $\mathrm{OH}$ mole fraction $\left(\mathrm{X}_{\mathrm{OH}}\right)$ and the critical stretch rate $(K)$ represents the production rate of $\mathrm{OH}$ radicals, i.e., the fuel reactivity. To account for the effect of fuel molecular transport and heating value, since the fuel diffusivity is proportional to the square root of molecular weight, the transport-weighted enthalpy (TWE, cal/ $\mathrm{cm}^{3}$ ) is defined as the fuel enthalpy scaled by the square root of the fuel molecular weight $(M W)[18]$;

$$
T W E=[\text { fuel }] \times \Delta H_{c} \times\left(M W_{\text {fuel }} / M W_{\text {nitrogen }}\right)^{-1 / 2}
$$


Here, [fuel] is the initial fuel concentration $\left(\mathrm{mol} / \mathrm{cm}^{3}\right)$ and $\Delta H_{c}$ is heat of combustion $(\mathrm{kcal} / \mathrm{mol})$. The dependence of the critical radius on the transport weighted enthalpy is plotted in Fig. 7 for both alkane and aromatic fuel mixtures. It is seen that for alkane fuels with similar fuel reactivity, the transport weighted enthalpy can correlate the critical radius well with the transport and the heating value. However, for aromatic fuels, the transport-weighted enthalpy alone is not able to correlate the critical radius. Therefore, an index of fuel reactivity is needed to correlate the critical radius for fuels with different fuel molecular structures and reactivity. Figure 8 shows the dependence of the critical radius on the product of premixed flame radical index and the transport weighted enthalpy. Despite the large difference in fuel molecular structure, size, and reactivity among the four fuels, the measured critical radius now collapses into one curve. The good correlation shows that the critical radius is governed by transport, heating value, and the fuel reactivity, and can be correlated using the fuel radical index and transport weighted enthalpy. In [18, 31], it was demonstrated that the radical index and transport-weighted enthalpy are successful metrics to correlate the diffusion flame extinction limits for different kinds of hydrocarbon fuels and methyl esters with a large range of molecular size and structure. A reasonable correlation of these two parameters with the critical radius shown in this paper suggests that the radical index and transport-weighted enthalpy may be good parameters to estimate the flame initiation process for alternative transportation fuels by examining the impact of fuel transport and reactivity on global combustion parameters.

\section{Conclusion}

The critical flame initiation radius for n-alkanes and aromatics with different molecular sizes and structures were measured. The results showed that the fuel transport properties, heating value, and fuel chemistry all affect the critical flame radius. The experimental results agree qualitatively with theory and numerical simulations. For lean mixtures of large n-alkanes with similar fuel reactivity, the results show that the critical radius is similar and decreases with increasing equivalence ratio. However, as the effective Lewis number decreases (equivalence ratio increases), the difference between the critical radius of the smaller alkane (n-heptane) and the larger alkane (n-decane) increases. For isomeric aromatic fuels, the results show that the fuel chemistry has a significant impact on the critical radius. Fuels with lower fuel reactivity (e.g. 1,3,5-trimethylbenzene) have a much larger critical radius. At atmospheric pressure, the results show that critical radius of lean aromatic mixtures is above $1 \mathrm{~cm}$, suggesting a large MIE requirement for ignition of lean mixtures at lower pressure. The comparison between numerical simulation and experiment shows that although simulations can predict the trend of the dependence of the critical radius on equivalence ratio and fuel molecular structure, there is a quantitative discrepancy between experiments and simulations. This is because the kinetic mechanisms are not validated against transient flame speed and extinction limit.

Two new premixed flame parameters, the radical index and transport-weighted enthalpy, are proposed to correlate the critical radius and advance understanding of the flame initiation process. The results show that although the transport-weighted enthalpy can correlate well with the critical radius of alkane fuels at different equivalence ratios, it cannot correlate the critical radius of aromatics with different fuel reactivity. By using the product of the premixed flame radical index and the transport-weighted enthalpy, the critical radius of all four tested fuels with different molecular structures, sizes, and reactivities at different equivalence ratios can be well correlated. The present results suggest that ignition failure in internal combustion engines and 
turbine engine relight can be reduced by varying the transport-weighted enthalpy, fuel reactivity, equivalence ratio, and pressure. Moreover, the radical index and transport-weighted enthalpy may be good parameters for screening alternative transportation fuels and examining the impact of fuel transport and reactivity to global combustion parameters.

\section{Acknowledgements}

This work is supported as part of the Combustion Energy Frontier Research Center, funded by the United States Department of Energy, office of Science, Office of Basic Energy Sciences under Award Number De-SC0001198 and the NSF grant CBET-1507358. 


\section{Figure Captions}

Figure 1. Schematic of experiment.

Figure 2. Comparisons of flame trajectories, changes of flame propagation speed $\left(\mathrm{S}_{\mathrm{b}}\right)$ with stretch rate $(\mathrm{K})$ for $(\mathrm{a}) \mathrm{nC10}$ and $\mathrm{nC} 7$ and for $(\mathrm{b}) \mathrm{nPB}$ and 135TMB.

Figure 3. Comparisons between the measured and numerically computed (a) critical radius and (b) critical stretch rate as a function of equivalence ratio for $\mathrm{nC} 10$ and $\mathrm{nC} 7$.

Figure 4. Comparisons between the measured and numerically computed (a) critical radius and (b) critical stretch rate at critical radius point in a function of equivalence ratio for nPB and 135TMB.

Figure 5. Comparisons of (a) flame propagation speed, (b) flame thickness, (c) maximum heat release rate (HRR), and (d) gross radical pool mole fraction among $\mathrm{nC} 10, \mathrm{nC} 7, \mathrm{nPB}$, and 135TMB from numerical calculations.

Figure 6. Computed maximum $\mathrm{OH}$ mole fractions at critical radius as a function of computed critical stretch rate among $\mathrm{nC} 10, \mathrm{nC} 7, \mathrm{nPB}$, and $135 \mathrm{TMB}$ at equivalence ratios from $0.7-1.0$.

Figure 7. Measured critical radius as a function of transport-weighted enthalpy for (a) nC10 and $\mathrm{nC}$, and (b) nPB and 135TMB.

Figure 8. Measured critical radius as a function of premixed flame radical index (PFRI) multiplied with transport-weighted enthalpy (TWE) for $\mathrm{nC} 10, \mathrm{nC} 7, \mathrm{nPB}$, and $135 \mathrm{TMB}$. 


\section{References}

[1] J.K. Lefkowitz, Y. Ju, R. Tsuruoka, Y. Ikeda, AIAA paper-2012-1133, (2012).

[2] T.-W. Lee, V. Jain, S. Kozola, Combustion and Flame, 125 (2001) 1320-1328.

[3] Y. Ju, K. Maruta, Progress in Energy and Combustion Science, 37 (2011) 669-715.

[4] M.B. Colket, S. Zeppierie, H. Hollick, S. Zhang, Fall Technical Meeting: Eastern States

Section of the Combustion Institute, (2011).

[5] Z. Chen, M. Burke, Y. Ju, Proceedings of the Combustion Institute, 32 (2009) 1253-1260.

[6] A.P. Kelley, G. Jomaas, C.K. Law, Combustion and Flame, 156 (2009) 1006-1013.

[7] D. Bradley, M. Lawes, M.S. Mansour, Combustion and Flame, 156 (2009) 1462-1470.

[8] H.H. Kim, S.H. Won, J.S. Santner, Z. Chen, Y. Ju, Proceedings of the Combustion Institute, 34 (2012).

[9] Z. Chen, in, Princeton University, 2008.

[10] Z. Chen, M.P. Burke, Y. Ju, Proceedings of the Combustion Institute, 33 (2011) 1219-1226.

[11] Z. Chen, Y. Ju, Combustion Theory and Modelling, 11 (2007) 427-453.

[12] M. Blanc, P. Guest, G. von Elbe, B. Lewis, The Journal of chemical physics, 15 (1947) 798802.

[13] J.-L. Beduneau, B. Kim, L. Zimmer, Y. Ikeda, Combustion and Flame, 132 (2003) 653-665. [14] J.K. Lefkowitz, P. Guo, T. Ombrello, S.H. Won, C.A. Stevens, J.L. Hoke, F. Schauer, Y. Ju, Combustion and Flame, 162 (2015) 2496-2507.

[15] S. Dooley, S.H. Won, M. Chaos, J. Heyne, Y. Ju, F.L. Dryer, K. Kumar, C.-J. Sung, H. Wang, M.A. Oehlschlaeger, R.J. Santoro, T.A. Litzinger, Combustion and Flame, 157 (2010) 2333-2339.

[16] S. Dooley, S.H. Won, J. Heyne, T.I. Farouk, Y. Ju, F.L. Dryer, K. Kumar, X. Hui, C.-J. Sung, H. Wang, M.A. Oehlschlaeger, V. Iyer, S. Iyer, T.A. Litzinger, R.J. Santoro, T.

Malewicki, K. Brezinsky, Combustion and Flame, 159 (2012) 1444-1466.

[17] S. Dooley, S.H. Won, S. Jahangirian, Y. Ju, F.L. Dryer, H. Wang, M.A. Oehlschlaeger, Combustion and Flame, 159 (2012) 3014-3020.

[18] S.H. Won, S. Dooley, F.L. Dryer, Y. Ju, Combustion and Flame, 159 (2012) 541-551.

[19] S.H. Won, S. Dooley, F.L. Dryer, Y. Ju, Proceedings of the Combustion Institute, 33 (2011) 1163-1170.

[20] P. Clavin, Progress in Energy and Combustion Science, 11 (1985) 1-59.

[21] S. Ishizuka, C.K. Law, in: Symposium (International) on combustion, Elsevier, 1982, pp. 327-335.

[22] F. Wu, W. Liang, Z. Chen, Y. Ju, C.K. Law, Proceedings of the Combustion Institute, 35 (2015) 663-670.

[23] M. Mehl, W.J. Pitz, C.K. Westbrook, H.J. Curran, Proceedings of the Combustion Institute, 33 (2011) 193-200.

[24] C.K. Westbrook, W.J. Pitz, O. Herbinet, H.J. Curran, E.J. Silke, Combustion and Flame, 156 (2009) 181-199.

[25] P. Diévart, H.H. Kim, S.H. Won, Y. Ju, S. Dooley, F.L. Dryer, W. Wang, M.A.

Oehlschlaeger, Fuel, (Accepted for Publication).

[26] W. Sun, X. Gou, Z. Chen, Y. Ju, Combustion and Flame, 157 (2010) 1298-1307.

[27] J.K. Bechtold, M. Matalon, Combustion and Flame, 127 (2001) 1906-1913.

[28] X. Hui, A.K. Das, K. Kumar, C.-J. Sung, S. Dooley, F.L. Dryer, Fuel, 97 (2012) 695-702.

[29] K. Kumar, C.-J. Sung, Combustion and Flame, 151 (2007) 209-224. 
[30] C. Ji, E. Dames, Y.L. Wang, H. Wang, F.N. Egolfopoulos, Combustion and Flame, 157 (2010) 277-287.

[31] P. Diévart, S.H. Won, J. Gong, S. Dooley, Y. Ju, Proceedings of the Combustion Institute, 34 (2013) 821-829. 